Meta

Journal des tradlucteurs

Translators' Journal

\title{
Mémoires en traduction déposés à l'Université de Montréal de 1955 à 1975
}

\section{Irène V. Spilka}

Volume 20, numéro 1, mars 1975

Vingt ans de traduction. Bilan et prospectives

URI : https://id.erudit.org/iderudit/004218ar

DOI : https://doi.org/10.7202/004218ar

Aller au sommaire du numéro

Éditeur(s)

Les Presses de l'Université de Montréal

\section{ISSN}

0026-0452 (imprimé)

1492-1421 (numérique)

Découvrir la revue

\section{Citer ce document}

Spilka, I. V. (1975). Mémoires en traduction déposés à l'Université de Montréal de 1955 à 1975. Meta, 20(1), 80-82. https://doi.org/10.7202/004218ar d'utilisation que vous pouvez consulter en ligne. 


\section{Mémoires en traduction déposés à l'Université de Montréal de 1955 à 1975}

LISTE THÉMATIQUE PAR ANNÉE

Théorie, méthode, problèmes de traduction

1961 : PRIEUR, Nicole, La traduction technique et administrative à l'O.A.C.I.

1967 : PÉLOQUIN, Pauline, La traduction du livret d'opéra.

1967 : CHARBONNEAU-DAGENAIS, Aline, Toponymie et traduction.

1971 : FORTIN, Suzanne, Problèmes de traduction médicale. Champ d'application : le diabète.

1971 : MUNTER, Yolaine, Etude statistique des différents procédés de traduction dans une publication gouvernementale: Rapport de la Commission royale d'enquête sur le bilinguisme et le biculturalisme.

1973 : FONTAINE, Jean-Paul, Humour et traduction.

1973 : GOYETTE LABERGE, Suzanne, Étude sur la traduction de quelques adjectifs (français-anglais).

Stylistique comparée, sémantique

1955 : FRANCCEUR, Marielle, La modulation en traduction.

1957 : BART, Gilbert, La transposition, principe de stylistique comparée.

1960: COUSINEAU-DESJARDIN, Andrée, Stylistique comparée du latin et du français.

1964 : STIPKOVIC, Nada, La théorie des lacunes en stylistique comparée.

1966: CHEVRETTE-ROBICHAUD, Claire, Les titres de romans : stylistique et traduction.

1966 : GRÉGOIRE, Claudette, Les lacunes de dérivation en français.

1967 : MORIN, Michelle, Aires sémantiques et stylistiques des faux amis du franco-canadien.

1970 : LANDREVILLE, Gaétan, Étude stylistique du lexique anglais-français des mammifères du Canada.

\section{Glossaires}

1956 : FRANCEEUR, Andrée, Étude lexicologique des instruments de musique. 1957: LAURENDEAU, Françoise, Vocabulaire de la publicité anglaise.

1963 : DIONNE, Claire, Los falsos gemelos del espanol.

1963 : LANDRIAULT-DUVAL, Estelle, Lexique comparé des sons et des bruits.

1966 : GRATTON-FREDETTE, Nicole, La glace, terminologie et stylistique. 
1968: METCALFE, Claire, Vocabulaire des conventions collectives de travail.

1968 : GAUTHIER-LAFONTAINE, Glossaire raisonné anglais-français des termes comptables.

1969 : BÉLANGER, Nycole, Le cheval (régions du corps-races-nature physiquemorphologie). Vocabulaire français-anglais / anglais-français.

1969 : HENRY, Ronald, La terminologie des titres et des grades dans l'enseignement français et anglais.

1970 : ARVANITAKIS JAREMA, Gonia, Étude comparative de la terminologie française et américaine des caractéropathies.

1970 : BELINGA, Jérôme, Glossaire raisonné de la langue des affaires étrangères (anglais-français).

1970 : MIGNAULT-VISSER, Lise, Vocabulaire des arts ménagers.

1971 : REGNIER-BELISLE, Gabrielle, Étude du vocabulaire de l'intervention sociale collective.

1971 : SANSREGRET, Louise, Glossaire raisonné anglais-français des termes de l'industrie du pétrole.

1971 : VIROLY, Michèle, Glossaire raisonné anglais-français de termes journalistiques.

1972 : ALAI-VIDAL, Ginous, Le cheval. Les robes et leurs particularités. Vocabulaire français-anglais et anglais-français.

1972 : BOYER, Jocelyne, Lexique trilingue des termes relatifs à la technique de la peinture en art.

1972 : GLEN, José, Vocabulaire de la plongée sous-marine.

1972 : LANGEVIN-THAUVETTE, Céline, Vocabulaire anglais-français des termes fondamentaux de la couture.

1972 : SCHWAB, Wallace, Lexique raisonné des lois de l'impôt sur le revenu au Canada.

1973 : FANDRICH-ZWYSSIG, Marie-Thérèse, Mobilier francais : $17^{\circ}$ et $18^{\circ}$ siècles.

1974 : ACHAKOBE, Eviano Ogheneruhro, Glossaire raisonné de la préparation du tabac (anglais-français).

1974 : OBAFEMI, Olajumoke Oladevo, Glossaire anglais-français de l'enfance inadaptée.

1974 : OKUWAKA, Godwin Onyekwuluje, Glossaire raisonné anglais-français des termes se rapportant à l'usage non médical des drogues.

Traductions d'œuvres

1966: HORGUELIN, Paul A., La première banque au Canada.

1970 : CARON, Marie, Traduction des Anti-Gallic Letters.

1970: CÔTÉ, Gérard, Traduction de Keeping your Balance in the Modern Church.

1971 : BERTRAND, Fancine, Les collectifs à la chasse.

1971: JURISIC, Vidosava, The Tito-Mihailoyitoh, an English translation of Yourichltch, Evgenije, Le Procès Tito-Mihailovitch.

1972 : JANSEN, Christianne, Histoire du matériel d'incendie au Canada. 
1974 : EDEM, Sam Joe, Traduction de Principes et techniques du placement en valeurs mobilières.

1974 : OGUNSANYA, Ebun Omowunmi, Traduction de les Camerounais occidentaux. La minorité dans un État bicommunautaire.

1974 : OSOBA, Adeyombo, Traduction de la Victime est-elle coupable?

1974 : UKONGA, Adenike, Traduction de les Relations humaines dans l'entreprise.

1974 : YOUNG, Folashade, Traduction de l'Archipel inachevé : culture et société aux Antilles françaises.

\section{Traductions comparées et critiques}

1965 : BEAULIEU, Oda-Josette, Marcel Aymé's, le Passe-muraille, a critical comparison of Wallace Fowlie's and Germaine Brée's translation.

1966 : HOUPERT-LENGELlÉ, Anne, le Petit Fût de G. de Maupassant étude critique de deux traductions.

1967 : CORMIER, France-Pauline, Les canadianismes et leurs correspondants anglais dans Maria Chapdelaine.

1971 : CLAXTON, Patricia, Canadian History Textbooks : A comparative Study by Marcel Trudel and Genevière Jain, Studies of the Royal Commission on Bilingualism and Biculturalism, $\mathrm{N}^{\circ} 5$, translated from the French.

1972 : CHEVALIER, Monique, Flowers for Algernon de Daniel Keyes : étude des niveaux dans la traduction de Roger Durand.

1972 : GABOURY-LADOUCEUR, Lorraine, Gynecological Diagnosis traduction anglaise de Diagnostic gynécologique.

1972 : LAMOUREUX-PEILA, Céline, The cricket on the Hearth: deux traductions.

Divers

1967 : SAINT-DENIS, Yvon, Etudes des anglicismes contenus dans une page de journal canadien français de 1890.

1965 : COLPRON, Gilles, Les anglicismes au Québec.

1966 : BAUDOIN, Anne-Marie, La voie moderne et la Traverse R.S.

1970: LABELLE, Guy, Étude de la langue d'affichage dans la région de Montréal.

1971 : CORIAT, Annie, Les interférences anglaises dans le français de Montréal. Étude stylistique de la langue de la publicité.

1971 : DESMARAIS, Lise, La traduction et l'enseignement du français, langue seconde. 